\title{
MULTI-FUNCTIONING AND MULTI-OPTIMISATION IN FEATHERS
}

\author{
S.C. BURGESS \\ Department of Mechanical Engineering, University of Bristol, Bristol, UK.
}

ABSTRACT

Natural organisms often have multiple functions and multi-optimisation in a single component or mechanism. In addition, natural organisms are highly integrated assemblies. In contrast, human design has traditionally avoided multi-functioning in single components because of the difficulties this presents in the design process. Only in recent years has there been a trend towards multi-functioning in engineering components. The advantage of multi-functioning is that extremely high levels of performance can be achieved. This paper gives examples of multi-functioning and multi-optimisation in a bird flight feather and a bird display feather. In each case, the advantages of multi-functioning are explained and analogies with man-made design are given.

Keywords: multi-functioning, multi-optimisation, shape factors.

\section{INTRODUCTION}

One of the interesting characteristics of natural organisms from a design point of view is the existence of multiple functions and multi-optimisation in a single component or mechanism. In addition, natural organisms are highly integrated assemblies with several sub-systems being closely integrated together. In contrast, human design has traditionally avoided multi-functioning in single components because of the difficulties this presents in the design process. Multi-functioning and multi-optimisation are very challenging because there are more constraints in the design process and therefore fewer possible solutions. In practice, multi-functioning leads to a need for very sophisticated design solutions.

When designing an engineering device, it has traditionally been recommended to design each component for one main function in order to make the behaviour of the device easier to understand and predict [1]. For example, in material selection methodology it has traditionally been assumed that components generally have one main function [2]. Another reason for avoiding multi-functioning in the past is the lack of multidisciplinary design teams [3] and a lack of suitable technology. Observations of past engineering devices show that they do indeed generally possess limited multi-functioning and integration of parts.

Only in recent years have engineers adopted a design philosophy of integrating different functions together in single components and mechanisms. For example, cars are becoming highly integrated, with computing hardware and software being closely integrated with mechanical sub-systems such as engines and braking systems.

Multi-functioning and integration have obvious benefits. The number of components in a device can be dramatically reduced and this can lead to compactness and low mass. Compactness and low mass can lead to many improved aspects of mechanical performance such as energy and space efficiency and speed of operation. Low part count can also lead to high levels of reliability and easier maintenance.

Integration and multi-functioning are very common in nature. Leonardo da Vinci was one of the first scientists to appreciate how the natural world contained optimal design. After studying many aspects of the natural world, Leonardo concluded: 'Although human genius through various inventions makes instruments corresponding to the same ends, it will never discover an invention more beautiful, nor more ready nor more economical than does nature, because in her inventions nothing is lacking, and nothing is superfluous' [4]. 
D'Arcy Thompson (1860-1948) was one of the first modern scientists to systematically study optimum design in nature. In 1917 he published his classic work On Growth and Form [5]. More recently, there has been a growing interest in optimum design in nature and its possible application to engineering design.

It is very useful to study multi-functioning and multi-optimisation in nature because lessons can be learnt about how to achieve these desirable attributes. This paper gives examples of multi-functioning and multi-optimisation in a bird flight feather and a bird display feather. In each case, the advantages of multiple functions are explained and analogies with man-made design are given.

\section{MULTI-FUNCTIONING IN BIRD FLIGHT FEATHERS}

The structure of a flight feather is shown in Fig. 1. There is a hierarchy of structures. The main feather stem comes first, then the barbs and finally the barbules. The stem has a massed array of barbs on each side that form the basic feather shape. Each barb itself has two sets of barbules. The barbules on one side have a set of hooks whilst the barbules on the other side are plain. Therefore, the hooked barbules can interlock with the plain barbules on the adjacent barb.

The flight feathers of birds can be considered to have three major functions: an aerodynamic function, a fail-safe function and a lightweight structural function. These functions are summarised in a function-means tree in Fig. 2. A function-means tree summarises the functions and solutions of a device at different levels of detail and shows where multi-functioning takes place. The functionmeans tree in Fig. 2 shows that certain features of the feather are optimal for more than one function. In particular, the hierarchical structure is optimal for all three functions and hence is a very important feature. Despite having three complex functions, the feather is a single integrated structure. The optimal design of a flight feather for these three major functions is described below.

\subsection{Optimal aerodynamic layout}

The overall asymmetric feather profile is optimal from an aerodynamic point of view because the barbs are very short on the leading edge and are therefore protected against buckling from the airflow. Another important aerodynamic feature is a one-way airflow mechanism at each barbule joint. The hooks and barbules are arranged so that they prevent air from going through them when the wing is pushed downwards, but they allow some air to pass through them when the wing is being pulled

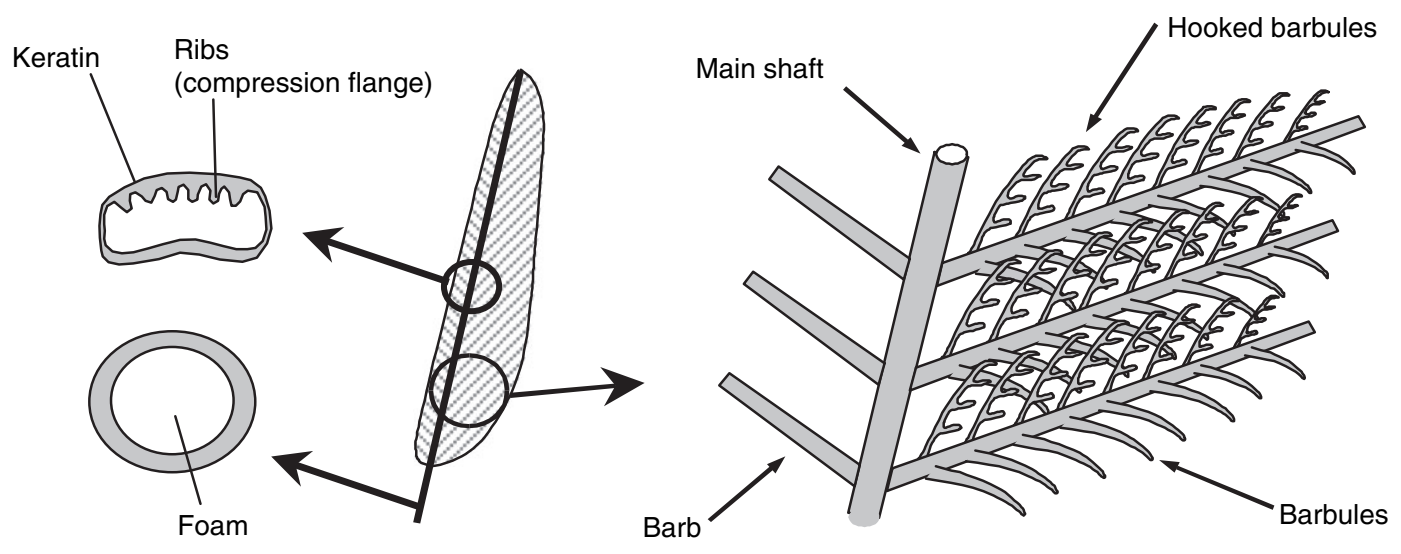

Figure 1: Structure of a flight feather. 
upwards [6]. This feature enables the bird to maximise the efficiency of flapping by making the wing mainly push the air down.

\subsection{Optimal fail-safe mechanism}

The flight feather of birds contains a localised fail-safe mechanism in the hook structure of the barbules. If the barbs are overloaded then they will unzip from adjacent barbs before any serious damage is done to the feather structure. Once unzipped, the barbs can be re-zipped together by the simple action of the bird passing its beak through the feather. The large number of separate zipping mechanisms ensures that the feather will unzip very close to the point of overload, thus causing minimum damage to the feather. The optimal fail-safe feature of the barbule connections leads to a high level of reliability in the wings of a bird [7].

\subsection{Optimal structural layout}

The hierarchical layout of the flight feather is optimal from a structural point of view because the feather transfers loads from a surface to a point. Bejan has shown that a hierarchical tree structure is generally the optimal solution for generating an efficient flow of forces (or heat or fluid) between a point source and a volume or surface [8]. The hierarchical structure of the feather is extremely important because it enables localised hooking mechanisms and fail-safe mechanisms as well as an optimum flow of forces.

As well as having an optimal hierarchical layout, the flight feather also has optimal material and shape properties. The feather consists of thin-walled keratin sections filled with lightweight foam.

\section{A BIRD FLIGHT FEATHER}

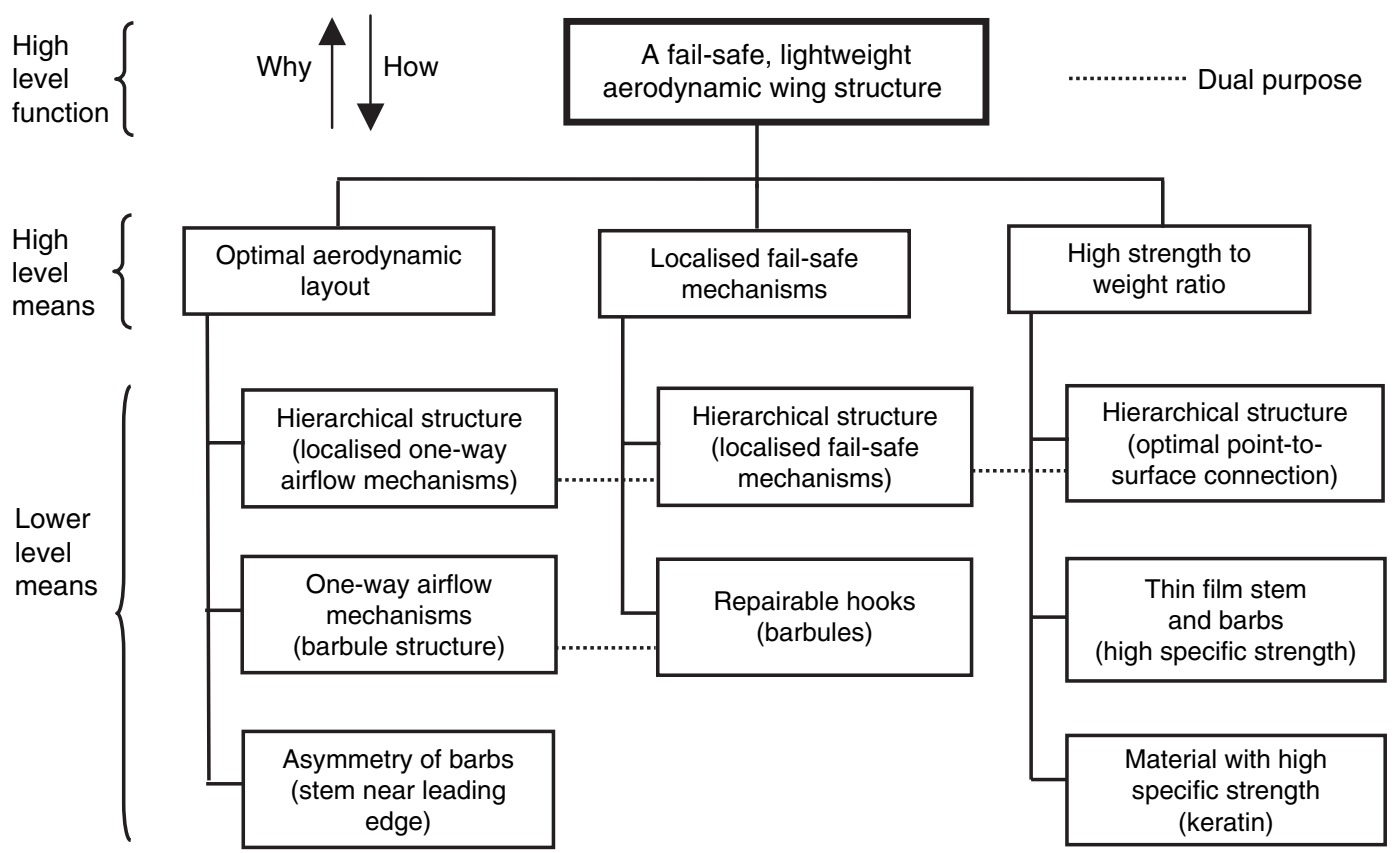

Figure 2: Function-means tree for a bird flight feather. 
Keratin has an extremely high specific strength. The shape is optimal for bending strength because the thin-walled sections place material away from the neutral axis. The cross-section of the stem starts off as circular near the root of the feather and then changes into a curved and ribbed rectangular shape away from the root. This arrangement is more structurally efficient for resisting bending and buckling. All of the structural sections are tapered so that the section size is well matched to the applied load.

It is possible to make a comparison between the structural efficiency of the feather stem and commonly available man-made tubular sections using the method of shape factors [9]. The following analysis compares the efficiency of a peacock flight feather stem with the highest performing steel and aluminium sections.

The mass of a beam of uniform section is given by:

$$
m=A l \rho,
$$

where $A$ is the cross-sectional area, $l$ is the length and $\rho$ is the density. Equation (1) can be solved for a particular shape of a beam by replacing the free variable $A$ with the strength requirement $M_{\mathrm{f}}$. The strength requirement is related to the beam shape by:

$$
M_{\mathrm{f}}=Z \sigma_{\mathrm{f}},
$$

where $M_{\mathrm{f}}$ is the failure moment, $Z$ is the section modulus and $\sigma_{\mathrm{f}}$ is the failure stress.

Since eqn (2) cannot be directly inserted into eqn (1), it is necessary to define a shape factor that relates the second moment of area to the cross-sectional area of the beam. In the case of dimensionally unconstrained beams, the shape factor is given by:

$$
\phi_{B}^{\mathrm{f}}=\frac{4 \pi^{1 / 2} Z}{A^{3 / 2}} .
$$

This shape factor is dimensionless and is defined such that it has a value of 1 for a solid circular shaft. Combining eqns (1)-(3) gives:

$$
m=\left(4 \pi^{1 / 2} M_{\mathrm{f}}\right)^{2 / 3} l\left(\frac{\rho^{2 / 3}}{\phi_{B}^{\mathrm{f}} \sigma_{\mathrm{f}}}\right)^{2 / 3} .
$$

Equation (4) defines the mass of a beam in terms of the functional requirements, the geometrical constraints and the material-shape properties. From eqn (4) it can be seen that the material-shape index can be defined as $M_{\mathrm{f}}=\left(\phi_{B(\max )}^{\mathrm{f}} \sigma_{\mathrm{f}}\right)^{2 / 3} / \rho$, which is inversely proportional to mass. An optimal material shape is therefore one that maximises $M_{\mathrm{f}}$.

Using eqn (3) the shape factor for a thin-walled beam is $(r / t)^{0.5}$, where $r$ is the radius and $t$ is the wall thickness [9]. A peacock flight feather was used to obtain the shape factor for a flight feather stem. The stem was measured to have a diameter of $7.5 \mathrm{~mm}$ and wall thickness of $0.2 \mathrm{~mm}$. This gives a shape factor of 4.3 for the flight feather.

Table 1 summarises the relative performance of steel, aluminium and keratin for tubular sections. The results show that keratin outperforms the best standard steel and aluminium sections by about 5 times. This means that the keratin circular section weighs less than a quarter of that of the steel and aluminium sections for a given bending strength requirement. This result demonstrates that the keratin feather is extremely efficient from a structural strength point of view, despite the fact that the feather performs three complex functions. 
Table 1: Performance of different materials for a given bending strength requirement.

\begin{tabular}{lcccc}
\hline Material & $\begin{array}{c}\text { Density } \\
\left(\mathrm{mg} / \mathrm{m}^{3}\right)\end{array}$ & $\begin{array}{c}\text { Minimum } \\
\text { strength, } \sigma_{\mathrm{f}} \\
(\mathrm{MPa})\end{array}$ & $\begin{array}{c}\text { Material-shape } \\
\text { factor, } \phi_{B(\max )}^{\mathrm{f}}\end{array}$ & $\begin{array}{c}\text { Material-shape index, } \\
M_{\mathrm{f}}=\left(\phi_{B(\max }^{\mathrm{f}} \sigma_{\mathrm{f}}\right)^{2 / 3} / \rho \\
\left(\mathrm{MPa}^{2 / 3} \mathrm{~m}^{3} / \mathrm{mg}\right)\end{array}$ \\
\hline Steel $^{*}$ & 7.9 & 500 & 5 & 23 \\
Aluminium $^{*}$ & 2.9 & 100 & 5.6 & 23 \\
Keratin $^{\dagger}$ & 0.8 & 200 & 4.3 & 114 \\
\hline
\end{tabular}

*Values of $\phi_{B(\max )}^{\mathrm{f}}$ for steel and aluminium were derived from reference [9].

${ }^{\dagger}$ Material properties for keratin were obtained from references [10] and [11].

\subsection{Engineering applications of multi-functioning structures}

An example of multi-functioning and multi-optimisation in structural engineering is the use of selfhealing mechanisms in composites. Researchers have devised a composite structure with embedded healing mechanisms that are activated when a crack develops [12]. The self-healing mechanism consists of distributed cells within the composite material that are activated to cure when the material is overstressed. The local cells are small enough so that the overall structural properties are not significantly compromised.

\subsection{Engineering applications of keratin}

Attempts are being made to exploit the properties of keratin by using it as a filler in plastics. Keratin is abundantly available in the chicken industry. At present feathers are either discarded or converted into a form of food. However, researchers have found ways of separating foam from keratin in order to produce pure keratin pieces for use as a filler. Tests have shown that the use of keratin has a beneficial effect on the mechanical properties of plastics [13].

\section{MULTI-FUNCTIONING IN BIRD DISPLAY FEATHERS}

Figure 3 shows a schematic representation of the peacock eye feather. It should be noted that a peacock is a male peafowl whereas a peahen is a female peafowl. The purpose of the peacock's feather is to form an attractive display during the courtship ritual in order to attract a peahen. The feathers are typically up to $1.5 \mathrm{~m}$ long and form a fan shaped display so that the feathers are clearly visible to the female. Each feather stands erect in isolation.

The display feathers have three main functions: a display colour function, a display layout function and a structural function. These functions are summarised in a function-means tree in Fig. 4. The function-means tree shows that certain features are optimal for more than one function. The feathers are highly optimised for both functions as described below.

\subsection{Optimal design of display colours}

The colours in a peacock's tail are not pigment colours but structural colours which are produced by an optical effect called thin-film interference [14]. The thin-film interference takes place in the transparent segments of the barbules and produces bright and iridescent colours. Iridescent colours are colours that have a deep lustre and change with the angle of view. 


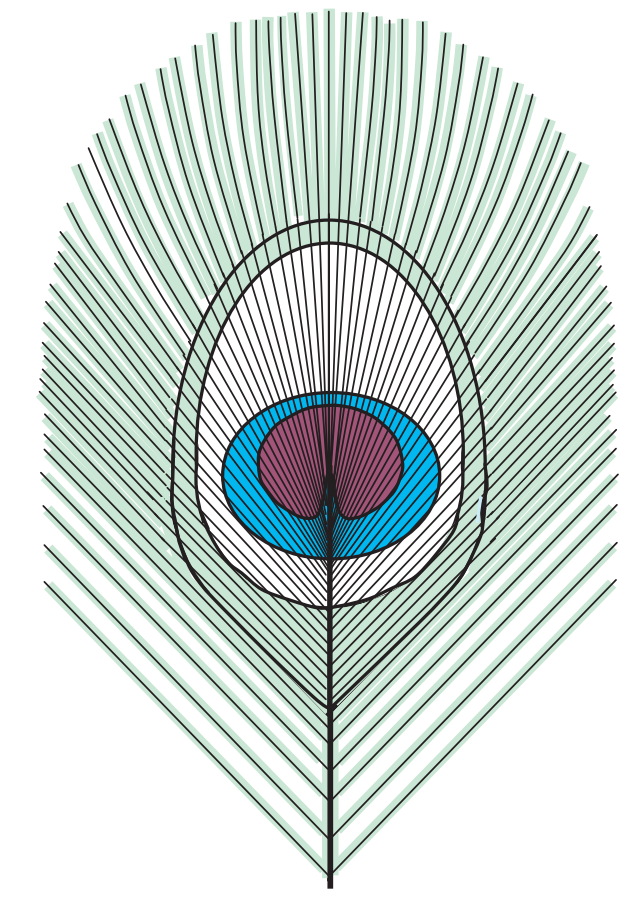

Figure 3: The peacock eye feather.

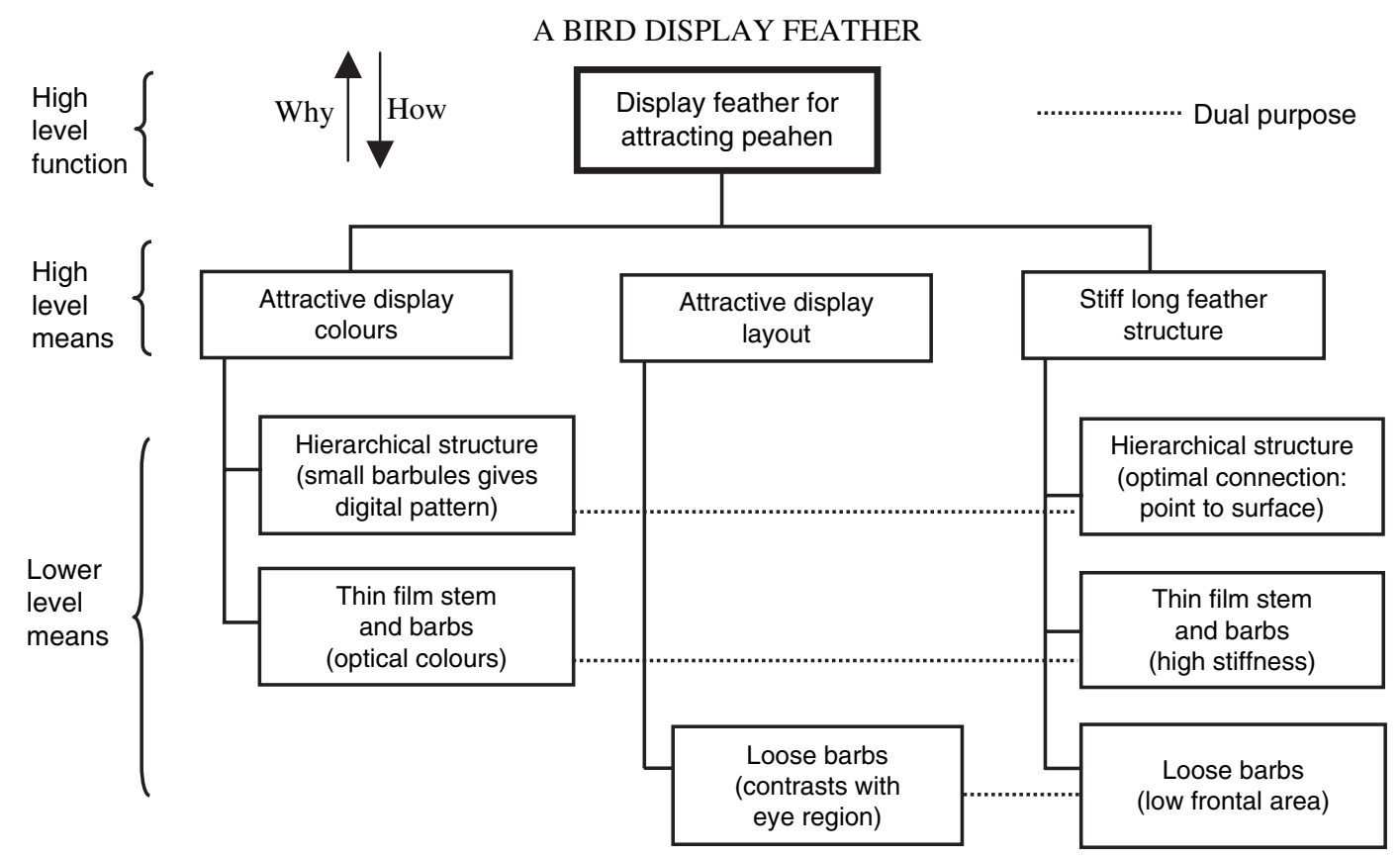

Figure 4: Function-means tree for a peacock display feather. 
Thin-film interference can be produced in one or more layers of a very thin and transparent material. The thickness of the transparent material must be close to the wavelengths of visible light. Visible colours have wavelengths of between 0.4 and $0.8 \mu \mathrm{m}$ and thin films typically have a thickness of between 0.3 and $1.5 \mu \mathrm{m}$. In the peacock tail feathers, thin-film interference takes place in three layers of keratin that surround the barbules. Each barbule is about $60 \mu \mathrm{m}$ wide and $5 \mu \mathrm{m}$ thick. The foam core is $2 \mu \mathrm{m}$ thick and the keratin layers are about $0.4-0.5 \mu \mathrm{m}$ thick [15].

The colours in the eye feather are produced when white light is reflected off the front and back surfaces of the keratin thin films. The light that passes through the keratin layer is retarded (slowed down) whereas the light that reflects off the front surface is not retarded. Therefore, some of the colour components of white light that are reflected from the back surface become out of phase with the corresponding light waves that are reflected off the front surface. When two wave trains of the same colour are out of phase, destructive interference takes place and the colour is removed. In the case of white light hitting thin films, as with a peacock's feathers, the result of the interference is a reflected colour due to the remaining colour components of white light.

The barbules in the peacock feather contain a remarkable degree of optimum design and produce bright colours with a deep lustre. The thickness of the keratin layers is optimal for producing the brightest thin-film colours. The three layers add to the brilliance of the colours in the feather by adding multiple components of light. A further optimum feature in thin-film design is that the barbules are slightly curved in the longitudinal direction. This curvature causes a mingling of slightly different colours and results in a softening of the colours seen in the keratin layers [15].

\subsection{Optimal design of display layout}

Figure 3 shows that the barbs under the eye pattern are loose which contrasts with the closely packed barbs in the eye pattern itself. The function-means tree shows that this is not just an aesthetic feature but also an optimal structural feature because it reduces wind loading by reducing the frontal area.

\subsection{Optimal structural design}

Like the flight feather, the display feather is highly optimised from a structural point of view with efficient materials, shapes and layout. Whereas the flight feather is designed mainly for strength, the display feather is designed mainly for stiffness because its function is to stand erect. A key design feature in the display feather is that the thin keratin layers are optimal not only from an optical interference point of view but also from a structural point of view.

As before, it is possible to make a comparison between the structural efficiency of the feather stem and commonly available man-made sections by using the method of shape factors [9]. The mass of a beam of uniform section is given by eqn (1). Equation (1) can be solved for a particular shape of a beam by replacing the free variable $A$ with the stiffness requirement $k$. The stiffness requirement is related to the beam shape by:

$$
k=C_{1} \frac{E I}{l^{3}},
$$

where $C_{1}$ is a constant that depends on the boundary conditions and loading of the beam, $E$ is the Young's modulus and $I$ is the second moment of area. For a cantilever, which is most common in nature, $C_{1}=3$. Since eqn (5) cannot be directly inserted into eqn (1), it is necessary to define a shape factor that relates the second moment of area $I$ to the cross-sectional area of the beam $A$. In the case 
Table 2: Performance of different materials for a given bending stiffness requirement.

\begin{tabular}{|c|c|c|c|c|}
\hline Material & $\begin{array}{l}\text { Density } \\
\left(\mathrm{mg} / \mathrm{m}^{3}\right)\end{array}$ & $\begin{array}{l}\text { Young's modulus, } \\
\qquad E(\mathrm{GPa})\end{array}$ & $\phi_{B(\max )}^{\mathrm{e}}$ & $\begin{array}{l}\text { Material-shape index, } \\
M_{\mathrm{e}}=\left(\phi_{B(\max )}^{\mathrm{e}} E\right)^{1 / 2} / \rho \\
\quad\left(\mathrm{GPa}^{1 / 2} \mathrm{~m}^{3} / \mathrm{mg}\right)\end{array}$ \\
\hline Steel* & 7.9 & 210 & 28 & 9.7 \\
\hline Aluminium* & 2.9 & 69 & 38 & 17.6 \\
\hline Keratin $^{\dagger}$ & 0.8 & 4 & 12 & 8.6 \\
\hline
\end{tabular}

*Shape factors for steel and aluminium were derived from reference [9].

${ }^{\dagger}$ Material properties for keratin were obtained from references [10] and [11].

of dimensionally unconstrained beams, the shape factor is given by:

$$
\phi_{B}^{\mathrm{e}}=\frac{4 \pi I}{A^{2}} .
$$

This shape factor is dimensionless and is defined such that it has a value of 1 for a solid circular shaft. Equation (6) shows that shapes with high values of $I / A^{2}$ have the highest shape factor. Combining eqns (1), (5) and (6) gives:

$$
m=\left(\frac{4 \pi l^{5} k}{C_{1}}\right)^{1 / 2}\left(\frac{\rho^{2}}{\phi_{B}^{\mathrm{e}} E}\right)^{1 / 2} .
$$

Equation (7) defines the mass of a beam in terms of the functional requirements, the geometrical constraint and the material-shape properties. Using eqn (6) the shape fact for a thin-walled rectangular section is given by:

$$
\phi_{B}^{\mathrm{e}}=\frac{\pi h(1+3 b / h)}{6 t(1+b / h)^{2}},
$$

where $h$ is the height, $b$ is the breadth and $t$ is the wall thickness. At one section, the stem of a peacock display feather was measured to have a breadth of $1 \mathrm{~mm}$, height of $2 \mathrm{~mm}$ and wall thickness of $0.1 \mathrm{~mm}$, thus giving a shape factor of approximately 12 for the section.

From eqn (7) it can be seen that the material-shape index can be defined as $M_{\mathrm{e}}=\left(\phi_{B(\max )}^{\mathrm{e}} E\right)^{1 / 2} / \rho$, which is inversely proportional to mass. An optimal material shape is therefore one that maximises $M_{\mathrm{e}}$. Table 2 summarises the relative performance of steel, aluminium and keratin for a thin-walled box section. The results show that the peacock feather has a similar performance to the best steel section but it is not as good as the best aluminium section. However, the result still shows that the feather has a very high specific stiffness.

\subsection{Multi-functioning in engineering}

The attractiveness of thin-film colours is such that they are now sometimes used on motorcar bodies as a key aesthetic feature. In automotive applications, small thin-film flakes are embedded in a binder and surrounded by pigment particles and metallic flakes [16]. Such paints have a dual functionality of providing a protective coating as well as a sophisticated aesthetic display. 


\section{CONCLUSIONS}

One of the main reasons for the exceptional performance and sophistication of bird feathers is the feature of multi-functioning. The design of bird feathers demonstrates that multi-functioning and multi-optimisation can produce large benefits in performance. Not surprisingly, there has been a recent trend in engineering design to move towards multi-functioning in single components and structures.

The hierarchical structure of feathers is one of the key features that enable multiple functions to be carried out in one integrated structure. The hierarchical structure enables fine-tuning of shapes and layout and enables a very large number of localised sub-mechanisms to exist. Hierarchical structures have been found to be important in other natural systems such as trees [17] and many other systems in nature [8].

Multi-functioning and multi-optimisation are very challenging because there are more constraints in the design process and hence fewer possible solutions. In addition, the design team must have wide cross-discipline knowledge to know what is feasible and optimal. Nature can be a rich source of ideas and inspiration that can help to achieve multi-functioning in engineering.

Multi-functioning in nature can be studied using methods such as function-means trees. Functionmeans trees are a good way of analysing multi-functioning because they help clarify aspects of the solution that are optimal for more than one function. Function-means trees are also good for considering functions of different disciplines such as industrial design (aesthetics) and engineering.

\section{REFERENCES}

[1] Pahl, G. \& Beitz, W., Engineering Design: A Systematic Approach, Springer-Verlag: London, p. 249, 1996.

[2] Ashby, M.F., Materials Selection in Mechanical Design, Pergamon Press: Oxford, 1992.

[3] Booker, J.D., Raines, M. \& Swift, K.G., Designing Capable and Reliable Products, Butterworth Heinemann: Oxford, p. 257, 2001.

[4] Thompson, B.S., Environmentally-sensitive design: Leonardo WAS right! Materials and Design, 20, pp. 23-30, 1999.

[5] Thompson, D., On Growth and Form, Cambridge University Press: Cambridge, 1961.

[6] King, A.S. \& McLelland, J., Birds-Their structure and Form, Bailliere Tindall: London, p. 30, 1984.

[7] Burgess, S.C., Reliability and safety strategies in living organisms: potential for biomimicking. Proceedings of the Institution of Mechanical Engineers. Part E: Journal of Process Engineering, 216, pp. 1-13, 2002.

[8] Bejan, A., Shape and Structure, from Engineering to Nature, Cambridge University Press: Cambridge, 2000.

[9] Weaver, P.M. \& Ashby, F., The optimal selection of material and section-shape. Journal of Engineering Design, 7(2), pp. 129-150, 1996.

[10] French, M., Invention and Evolution, 2nd edn, Cambridge University Press: Cambridge, p. 63, 1994.

[11] Vogel, S., Comparative Biomechanics, Princeton University Press: Princeton, NJ, pp. 310-312, 2003.

[12] Pang, J.W.C. \& Bond, I.P., 'Bleeding composites'-damage detection and self-repair using a biomimetic approach. Composites Part A: Applied Science and Manufacturing, 36(2), pp. 183-188, 2004.

[13] Schmidt, W.F., Polymer composite keratin, US Patent No. SN 10/750.464, 31 December 2003. 
[14] Zi, J., Yu, X., Li, Y., Hu, X., Xu, C., Wang, X., Liu, X. \& Fu, R., Colouration strategies in peacock feathers. Proceedings of the National Academy of Sciences, 100(22), pp. 12576-12578, 2003.

[15] Mason, C.W., Structural colours in feathers II. Journal of Physical Chemistry, 27, pp. 416-417, 1923.

[16] Ershov, S., Kolchin, K. \& Myszkowski, K., Rendering pearlescent appearance based on paint-composition modelling. Computer Graphics Forum, 20(3), 2001.

[17] Burgess, S.C. \& Pasini, D., An analysis of the structural efficiency of trees. Journal of Engineering Design, 15(2), pp. 177-193, 2004. 